\title{
Large Gathering Attendance is Associated with Increased Odds of Contracting COVID-19: A Survey Based Study
}

\author{
Mary E. Schroeder ${ }^{1}$ (D) Michelle TB Manderski ${ }^{2} \cdot$ Chris Amro $^{4}$. \\ Sneha Swaminathan ${ }^{3}$. Akshat Parekh ${ }^{3}$. Sho Yoshitake ${ }^{3}$. Jason Yang ${ }^{3}$. \\ Paul Romeo $^{3} \cdot$ Daniel Reyes ${ }^{3} \cdot$ Rachel Choron $^{4} \cdot$ Michael Rodricks $^{4}$
}

Accepted: 18 November 2021 / Published online: 6 January 2022

(c) The Author(s), under exclusive licence to Springer Science+Business Media, LLC, part of Springer Nature 2022, corrected publication 2022

\begin{abstract}
We used a telephone survey to determine risk factors associated with a positive polymerase chain reaction test of a nasopharyngeal swab for severe acute respiratory syndrome coronavirus 2 (SARS-Cov-2) at a community hospital in Central New Jersey during the early stages of the pandemic. We compared survey responses of 176 patients in March 2020. Respondents were asked about their living situation, work environment, use of public transportation and attendance at one or more large gatherings (more than 10 people) in the 3 weeks prior to undergoing COVID testing. We found that those who attended a large gathering in the 3 weeks prior to their COVID test had a 2.50 odds ratio $(95 \%$ CI $1.19,5.22)$ of testing positive after controlling for age, sex, race/ethnicity, occupation, living situation and recent visit to a nursing home. The total number of gatherings attended or the number of people in attendance was not associated with a positive test. An association was also seen for specific job types such as factory workers, construction workers, and facilities managers. Attendance at a gathering of more than ten people was associated with testing positive for COVID-19.
\end{abstract}

Keywords COVID-19 · Transmission · Prevention · Survey · Large gathering

Mary E. Schroeder

meschroeder@mcw.edu

1 Division of Trauma and Acute Care Surgery, Medical College of Wisconsin, 8701 West Watertown Plank Road, 53226 Milwaukee, WI, United States

2 Department of Biostatistics and Epidemiology, Rutgers University School of Public Health, Piscataway, NJ, United States

3 Rutgers Robert Wood Johnson Medical School, New Brunswick, NJ, United States

4 Division of Acute Care Surgery, Rutgers Robert Wood Johnson Medical School, New Brunswick, NJ, United States 


\section{Introduction}

Severe acute respiratory syndrome coronavirus 2 (SARS-CoV-2) was first identified in Wuhan, China in December 2019. Over the next several months Coronavirus Disease 2019 (COVID-19) cases were reported in over one hundred countries with the number of cases in the United States increasing from 30 to 213,144 in March 2020 (Centers for Disease Control, 2020). The first case of COVID-19 in New Jersey was reported March 4th (Dominus, 2020), and by April 1st the state reported a total of over 22,000 confirmed cases (Nart, 2020).

As this virus emerged and hospitals in New Jersey were inundated with patients, the risk factors associated with transmission were unclear. State level public health initiatives were implemented, including school closures beginning March 18th, 2020 and stay-at-home orders March 21st, 2020 (Office of the Governor of New Jersey, 2020). Despite these initiatives, the trajectory of new cases in New Jersey continued unabated in March and April.

In this study, we sought to determine the risk factors associated with testing positive for COVID-19 at a mid-sized community hospital in Central New Jersey during March 2020.

\section{Methods}

The study was performed at a 297-bed community hospital. After obtaining Institutional Review Board approval from Somerset Hospital, the principal investigator obtained a list of all patients that received a COVID-19 test at the hospital. COVID-19 testing was performed utilizing a polymerase chain reaction test of a nasopharyngeal sample.

Demographic information as well as history of comorbidities was extracted from the electronic medical record (Allscripts-Sunrise Clinical Manager, Chicago, IL). Additional data collected included admission status, hospital length stay, intensive care unit (ICU) admission, ventilator days, mortality, and contact information for the patient. Patients who presented from a nursing home, rehabilitation center, or group home were excluded from the survey as the group living arrangement served as a risk factor for transmission of the virus that would bias the survey data.

A maximum of three attempts was made on three separate days to contact each patient, or their next of kin, via telephone. Participants could complete the survey in English or Spanish, administered by a native speaker to ensure response fidelity. Participants provided verbal consent for the survey. The survey took approximately 10 min to complete and consisted of 13 questions with response options as seen in Table 1; branched logic was used as appropriate. Answers were verified with the respondent. A large gathering was defined as more than 10 people. The next of kin contacted were asked to complete the survey to the best of their ability for patients that had died of COVID-19. We collected all data from the medical record and entered the survey into a REDCap database (Vanderbilt University, Nashville, TN). 
Table 1 Survey questions and response options (in parentheses)

1. Prior to your hospital presentation, what was your living situation? (Lived alone, lived with roommates, lived in a group home, lived in a nursing home, lived in a rehab, other)

2. How many people lived with you?

3. Did any of them show signs of infection prior to admission?

4. At the time of your presentation, did you work?

5. What type of work setting? (Office, School/child care, factory/warehouse, construction/landscaping, facilities management, restaurant/food industry, retail, other)

6. Approximately how many people do you work with?

7. Was anybody at work sick prior to your presentation to Somerset Hospital?

8. Do you commute to New York City regularly?

9. Do you use public transportation? If yes, what type? (Train, bus, other)

10. In the 3 weeks prior to your presentation to Somerset did you visit a nursing home, hospital or longterm care facility?

11. Approximately how many of these events did you attend?

12. Approximately how many people were in attendance?

13. Have you identified any other places/events where you think you may have been exposed to coronavirus? If yes please provided details.

Descriptive statistics were collected and summarized to describe the study population. We reported median and interquartile range (IQR) for continuous variables, as most were non-normally distributed according to the Shapiro-Wilk statistic. We compared categorical variables using Pearson's Chi-Square or Fisher's Exact test when warranted due to small sample size. Continuous variables were compared using 2-sided Wilcoxon Rank-Sum test. We performed multivariable logistic regression analyses to estimate the strength of association between attendance at a large gathering within the prior three weeks and COVID-19 test result, adjusted for age, gender, and race/ethnicity (Model 1); plus recent visit to a nursing home, hospital or long term care facility within the prior 3 weeks (Model 2); plus living situation and work setting (Model 3); plus history of chronic respiratory disease (Model 4). We selected regression covariates based upon prior theory and results of the bivariate comparisons. We performed two additional regression analyses, restricted to respondents who attended any large gathering, to assess whether number of gatherings attended or number of people in attendance was associated with odds of a positive COVID-19 test. Finally, We compared patient characteristics for study participants versus non-participants. We conducted all analyses using SAS Version 9.4 (SAS Institute, Cary, NC).

\section{Results}

A total of 399 patients were tested for COVID-19 in March at a community hospital; 176 participated in the telephone survey, giving a $44 \%$ response rate. One-hundredand-six survey participants were COVID-positive (60\%) while 70 participants tested negative. 
Based on the medical records, COVID-positive survey respondents, relative to COVID-negative respondents, were older (median age, 56.5 vs. $45.0, p<.001$ ), more likely to be male $(54.7 \%$ vs. $41.4 \%, p=.08)$, admitted to the hospital $(52.4 \%$ vs. $22.4 \%, p<.001)$, admitted to the ICU $(18.1 \%$ vs. $3.0 \%, p=.003)$, and intubated $(16.2 \%$ vs. $1.5 \%, p=.002)$. History of chronic respiratory disease was less prevalent among COVID-positive respondents than COVID-negative respondents $(5.7 \%$ vs. $27.3 \%, p<.001)$. COVID-19 status did not significantly differ with the prevalence of hypertension, diabetes, smoking, and obesity. COVID-19 status did not significantly differ with racial/ethnic distributions except for non-Hispanic Asians for whom a higher proportion were COVID-positive (26.4\% vs. $17.1 \%$ for COVID-negative respondents).

Analysis of survey data (see Table 1) found no association between living situation and COVID-19 test result. COVID-19 status did not significantly differ with employment. However among employed respondents, more COVID-positive than COVID-negative participants worked in a factory, construction, or facilities setting while COVID-negative participants were more likely to work in healthcare settings. COVID-19 status did not correlate with number of co-workers. The proportions of COVID-positive and COVID-negative respondents who commuted to New York City were both low. Having visited a nursing home within three weeks prior to presentation was significantly less prevalent among COVID-positive respondents, whereas having attended a large gathering was more prevalent among COVID-positive respondents.

In multivariable regression (see Table 2), the magnitude of association between attendance at a large gathering and COVID test result increased when adjusting for visiting a nursing home, living situation, and occupation in addition to age, sex, and race/ethnicity. Among respondents who reportedly attended at least one large gathering, odds of a positive COVID-19 test did not differ according to the number of gatherings attended (per 1 additional gathering, OR $0.96,95 \%$ CI $0.86,1.06$ ) or the number of people in attendance at the gatherings (per 10 additional people, $O R$ $1.0005,95 \%$ CI 1.00, 1.00) (Table 3).

\section{Discussion}

We found that attending even a single large gathering was consistently and positively associated with a positive COVID-19 test. Notably, the odds of a positive test did not significantly increase with the number of people in attendance or the number of events attended by the survey respondent. A single event was enough to increase odds of contracting COVID-19.

We hypothesize that, unlike daily or weekly tasks such as grocery shopping, large gatherings result in stationary positions for longer periods of time, creating longer exposures and higher risk of virus transmission. As businesses reopen and "normal" activities resume, individuals must weigh the risks and benefits of each outing as a potential opportunity for exposure. Based on our study, attendance at a single large gathering such as a movie, church service, party, or sporting event is associated with an increased risk of contracting COVID-19. 


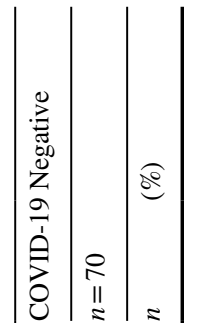

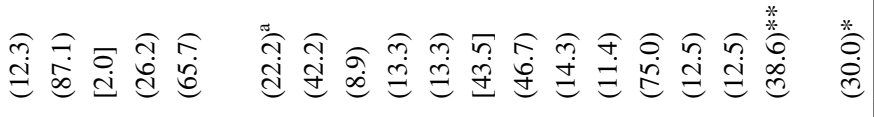
in

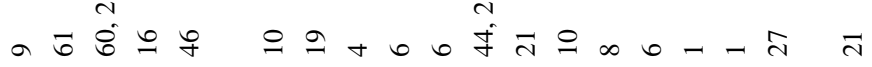

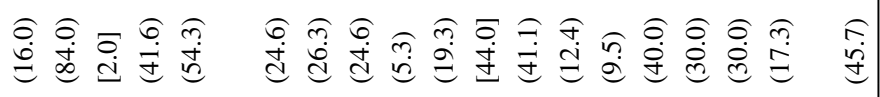
i.

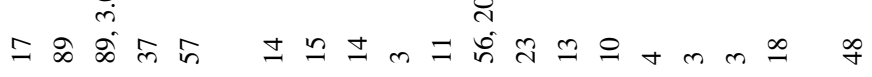

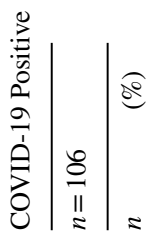

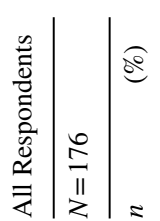

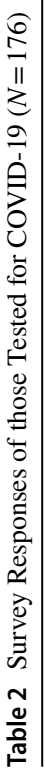

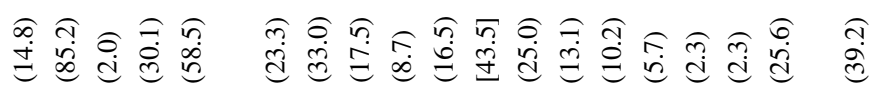

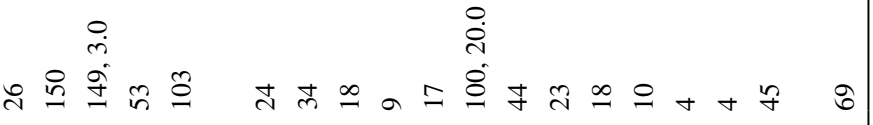




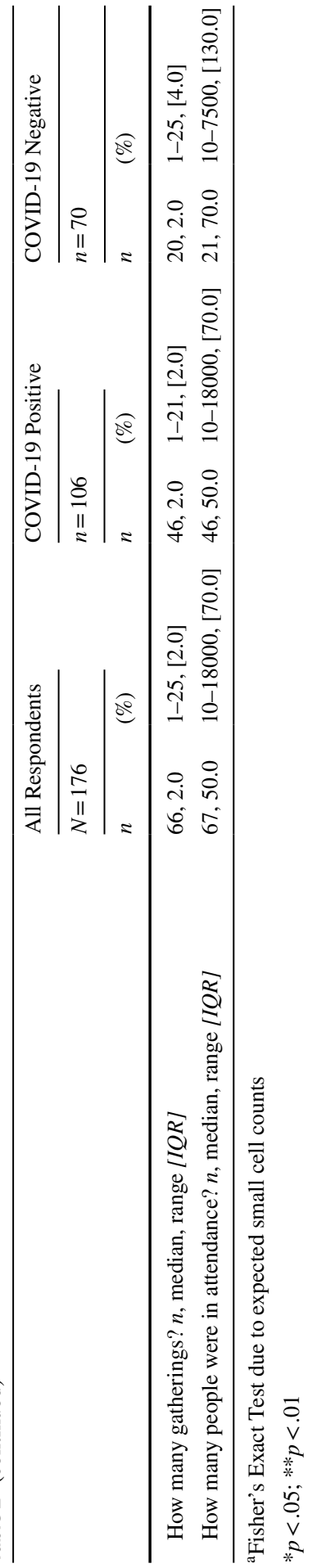




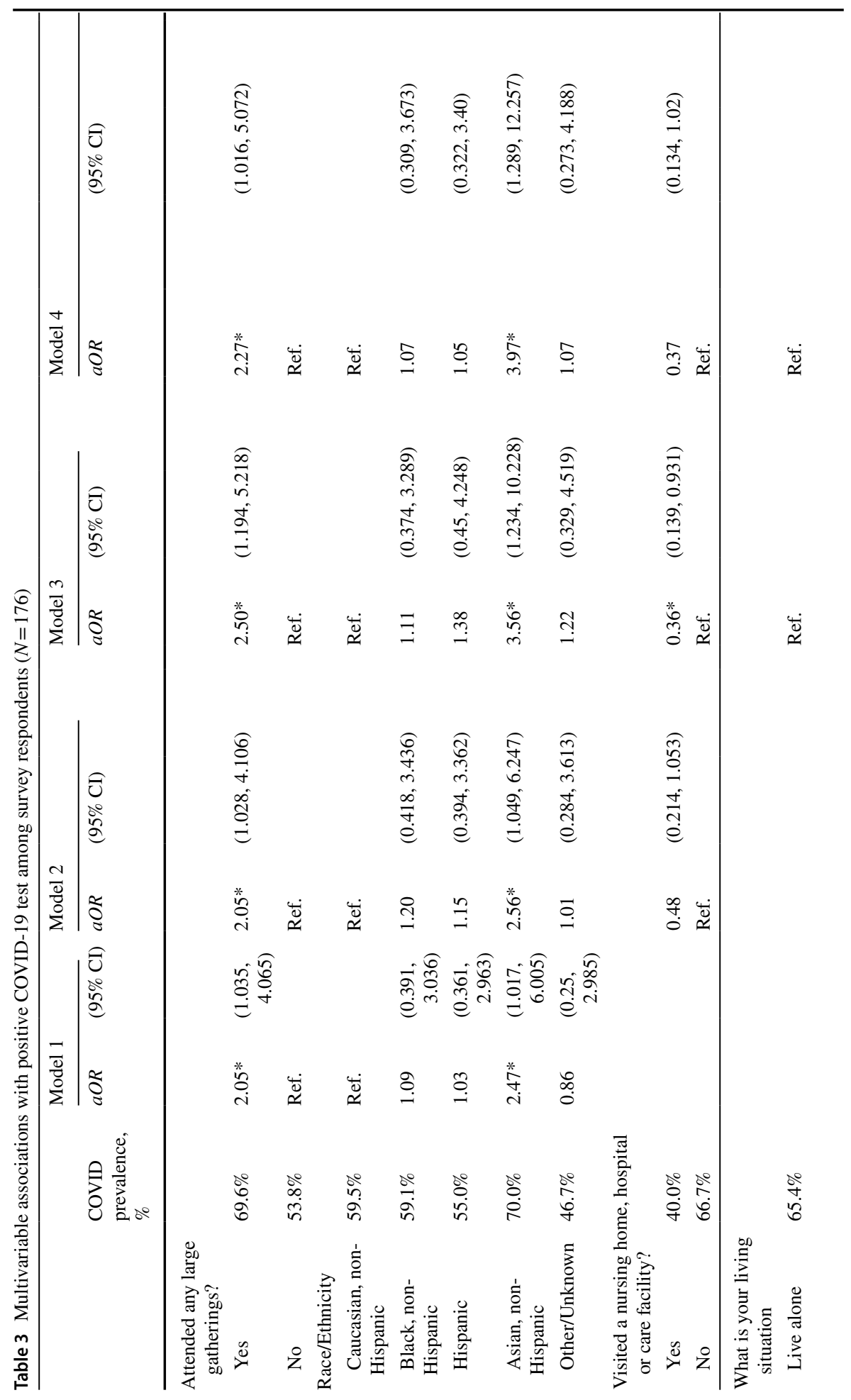




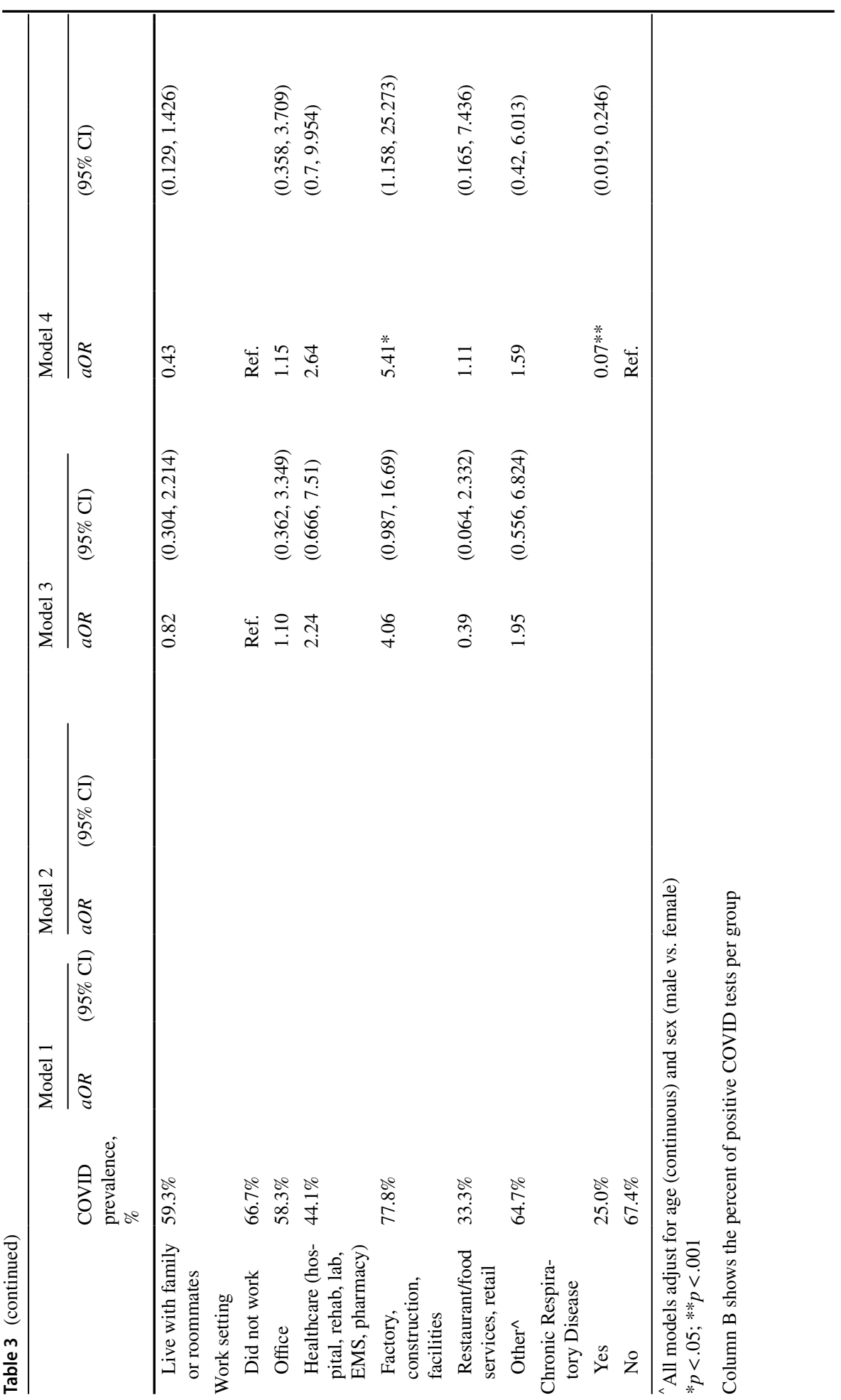


Regarding occupation, survey respondents who worked in a factory, construction, or facilities (i.e., environmental or janitorial services) setting had significantly higher odds of a positive test compared to those who were unemployed. Potential factors influencing this finding in the industrial setting include lower temperatures facilitating transmission, droplet exposure, and proximity to coworkers (Middleton, Reintjes, \& Lopes, 2020). These factors also explain why other job settings of similar socioeconomic status, such as food services and retail, do not exhibit the same increase in odds of a positive test.

Our survey shows that respondents that worked in health care were more likely to be COVID-19 negative. While this initially seems counterintuitive, we hypothesize that this is related to high rates of concern for exposure amongst those in health care and access to testing through the workplace. Of note, we did not specifically ask health care workers if they were working with COVID-19 patients.

Our study took place in the early days of the pandemic's spread, a time when little about the virus was well-understood and before mask mandates were in place. In the subsequent year, researchers have worked diligently to identify the risks associated with various daily activities (Sun et al., 2020) as well as the impact of social distancing and shelter-in-place policies (Cobb, 2020; Lyu, 2020). Our study adds to this body of work, making the case for restriction of large gatherings as a means to mitigate spread of SARS-CoV-2.

The limitations of this study should be noted. The retrospective nature of this survey makes it subject to recall bias. We also surveyed only those that were tested for COVID in a hospital setting, rather than a survey of the general population, leading to selection bias. In addition, the next of kin were surveyed in the case of a patient's death, introducing further recall bias. Participants were also more likely than nonparticipants to have tested positive for COVID-19, suggesting our study population may not be representative of all patients, and that possibly the severity of symptoms led these participants to the hospital setting. However, we did not observe significant differences in demographics or comorbidities when comparing participants to nonparticipants, tempering concerns about selection bias. In addition, the use of PPE was not asked of survey respondents as the national guidance at that time, March 2020, was to preserve and reallocate PPE to healthcare workers.

\section{Conclusions}

Our results indicate that gathering in groups as well as factory, construction, or facilities job settings are modifiable risks factors associated with positive COVID-19 test results in March 2020 at our community hospital in Central New Jersey. It is important that, as states move towards reopening, public officials be aware of what types of events carry the highest risk to citizens and, therefore, weigh the risks and benefits of each sequential step towards returning to normal. While vaccination may solve many of these concerns it is assumed that there will be future pandemics for which state and federal governments must be prepared. A better understanding of relative risks of various 
behaviors may help enact policies that allow for judicious restriction of activities while preventing a shutdown as has been seen during this pandemic

Authors' Contribution: All authors contributed substantially to the manuscript. Their contributions are listed below, based on CRediT taxonomy: Michelle TB Manderski PhD, MPH: Methodology, formal analysis, data curation, writing original draft. Chris Amro MD: Methodology, investigation, writing-review and editing, supervision. Sneha Swaminathan: Methodology, investigation, writing original draft. Akshat Parekh: Methodology, investigation, writing - review and editing. Sho Yoshikate: Methodology, investigation, writing — review and editing. Jason Yang: Methodology, investigation, writing — review and editing. Paul Romeo: Methodology, investigation, writing - review and editing. Daniel Reyes: Methodology, investigation, writing-review and editing. Rachel Choron MD: Conceptualization, writing-review and editing. Michael Rodricks MD: Methodology, conceptualization, writing original draft. Large gathering attendance is associated with increased odds of contracting COVID-19: a survey based study.

\section{Declarations}

Disclosures The authors have no relevant financial or non-financial interests to disclose.

Conflicts of Interest The authors have no conflicts of interest or financial disclosures to report.

\section{References}

Centers for Disease Control (2020). Provisional COVID-19 Death Counts by Week Ending Date and State. https://data.cdc.gov/NCHS/Provisional-COVID-19-Death-Counts-by-Week-Ending-D/r8kw-7aab. Published May 2020. Accessed June 20, 2020

Cobb, J., \& Seale, M. (2020). Examining the effect of social distancing on the compound growth rate of COVID-19 at the County level (United States) using statistical analyses and a random forest machine learning model. Public Health, 185, 27-29. https://doi.org/10.1016/j.puhe.2020.04.016

Dominus, S. (2020). How New Jersey's first coronavirus patient survived. New York Times website. https:// www.nytimes.com/2020/04/05/magazine/first-coronavirus-patient-new-jersey.html. Published April 5, 2020. Accessed June 20, 2020

Lyu, W., \& Wehby, G. L. (2020). Shelter-in-Place orders reduced COVID-19 mortality and reduced the rate of growth in hospitalizations. Health Affairs, 39(9), 1615-1623. https://doi.org/10.1377/hlthaff.2020. 00719

Middleton, J., Reintjes, R., \& Lopes, H. (2020). Meat plants-a new front line in the COVID-19 pandemic. $B M J, \mathrm{~m} 2716$. https://doi.org/10.1136/bmj.m2716

Nart, J. (2020). COVID19-Trend Tracker. https://public.tableau.com/profile/jonas.nart?fbclid=IwAR0zhzuZ qh5pD_rb3NDvVKNtYurbS-6EFYvQRS6RMVZSbVjMvQkdacUWP2k\#!/vizhome/COVID19_ 15844962693420/COVID19-TrendTracker. Published March 17, 2020. Accessed June 20, 2020

New Jersey COVID-19 Information Hub (2020). How can people safely get together? What are the limits for indoor and outdoor gatherins? https://covid19.nj.gov/faqs/nj-information/reopening-guidance-andrestrictions/how-can-people-safely-get-together-what-are-the-limits-for-indoor-and-outdoor-gatherings\# direct-link. Accessed June 20, 2020

Office of the Governor of New Jersey (2020). Governor Murphy announces statewide stay at home order, closure of all non-essential retail businesses. https://www.nj.gov/governor/news/news/562020/appro ved/20200320j.shtml. Published March 21, 2020. Accessed June 20, 2020

Sun, Z., Di, L., Sprigg, W., Tong, D., \& Casal, M. (2020). Community venue exposure risk estimator for the COVID-19 pandemic. Health \& Place, 66, 102450. https://doi.org/10.1016/j.healthplace.2020.102450

Publisher's Note Springer Nature remains neutral with regard to jurisdictional claims in published maps and institutional affiliations. 